\title{
Synthesis and Characterization of Graphene Oxide Derivatives via Functionalization Reaction with Hexamethylene Diisocyanate ${ }^{\dagger}$
}

\author{
José A. Luceño-Sánchez 1, Georgiana Maties 2, Camino Gonzalez-Arellano ${ }^{2}$ and \\ Ana M. Díez-Pascual 1,* \\ 1 Departmento de Química Analítica, Química Física e Ingeniería Química, Facultad de Ciencias, \\ University of Alcalá, E-28871 Madrid, Spain; jose.luceno@uah.es \\ 2 Departmento de Química Orgánica y Química Inorgánica, Facultad de Ciencias, University of Alcalá, \\ E-28871, Madrid, Spain; georgiamaties@gmail.com (G.M.); camino.gonzalez@uah.es (C.G.-A.) \\ * Correspondence: am.diez@uah.es; Tel.: +34-918-856-430 \\ + Presented at the 1st International Online Conference on Nanomaterials, 1-15 September 2018; Available \\ online: https://iocn-2018-1.sciforum.net/.
}

Published: 30 August 2018

\begin{abstract}
Graphene oxide (GO), the oxidized form of graphene, shows unique properties, such as strong mechanical strength, high thermal conductivity, amphiphilicity, and surface functionalization capability that make it very attractive in various fields, ranging from medicine to optoelectronic devices and solar cells. However, its insolubility in non-polar and polar aprotic solvents hinders some applications. To solve this issue, novel functionalization strategies are pursued. In this regard, the current study deals with the preparation and characterization of hexamethylene diisocyanate (HDI)-functionalized GO. Different reaction conditions were tested to optimize the functionalization degree (FD), and detailed characterization was conducted via Fourier-transformed infrared (FT-IR) spectroscopy to confirm the success of the functionalization reaction. The HDI-GO could further react with other organic molecules or polymers via the remaining oxygen groups, which makes them ideal candidates as nanofillers for high-performance GO-based polymer nanocomposites.
\end{abstract}

Keywords: graphene oxide; hexamethylene diisocyanate; functionalization degree; solubility; infrared spectroscopy

\section{Introduction}

Graphene (G), an allotrope of carbon like diamond, graphite, and fullerenes, has attracted a lot interest in recent years both for fundamental studies and potential applications [1]. It is a flat, atomically thick, 2D sheet composed of $\mathrm{sp}^{2}$ carbon atoms arranged in a honeycomb structure. It presents superior electronic, thermal, and mechanical properties, a very large surface area, and the highest electrical conductivity known at room temperature [2]. It has an extremely high carrier mobility, broad absorption spectral range, high optical transparency, and its abundance make it a very attractive material in various fields ranging from medicine [3] or high-performance composites [4], to chemical sensors [5] and solar cells [6].

Several routes have been reported in the preparation of $G$, including chemical vapor deposition (CVD) of hydrocarbons onto transition metal surfaces, micromechanical exfoliation of graphite, epitaxial growth on electrically insulating substrates, like $\mathrm{SiC}$ wafers, electrochemical intercalation, thermal exfoliation, or chemical reduction of graphite oxide [7]. However, these approaches lead to a low production yield and are time consuming. Further, G is insoluble in common organic solvents, 
which hinders its applications. In this context, graphene oxide (GO), originated from the exfoliation of graphite oxide or the chemical oxidation of G [8], has been studied in many researches as an alternative to $\mathrm{G}$. The established advantages of GO in production yield and cost, make it an attractive candidate as a nano-filler in polymer composites. $\mathrm{GO}$ is a water-soluble nanomaterial that comprises epoxide, hydroxyl, and carbonyl groups on the basal planes and carboxylic acids on the edges. Thus, upon sonication in aqueous media, it easily exfoliates and forms a stable colloidal suspension due to its strong hydrophilicity [9]. However, the exfoliation of GO in organic solvents is hindered due to the strong hydrogen bonding interactions between the adjacent GO layers, which prevent the penetration of organic molecules into the interlayer spaces. The hydrophilicity of GO has been regarded as a drawback to develop high-performance polymer/GO nanocomposites in organic solvents. To solve this issue, novel functionalization strategies are pursued. An interesting approach is the isocyanate functionalization proposed by Stankovich et al. [10]. In their work, reactions between different organic isocyanates, and the hydroxyl and carboxyl groups of GO, were tested to reduce the amount of hydrogen bonds with donor groups on GO sheets, thus reducing the nanomaterial hydrophilicity. As a result, the isocyanate-treated GO samples could be exfoliated in some polar aprotic solvents such as $\mathrm{N}, \mathrm{N}$-dimethylformamide (DMF), after a mild ultra-sonication. However, they could not be dispersed in non-polar solvents, and this still restricts its use in certain applications, like polymeric-based solar cells.

In this regard, the present work focuses on the synthesis and characterization of hexamethylene diisocyanate (HDI)-functionalized GO. In our approach, GO was first prepared using a modified Hummers' method from flake graphite [8], and then reacted with HDI in the presence of triethylamine (TEA) as a catalyst to yield functionalized HDI-GO nanosheets (Scheme 1). Different reaction conditions were tested in order to optimize the functionalization degree (FD), and characterization was conducted to confirm the successful functionalization of HDI on the GO surface.
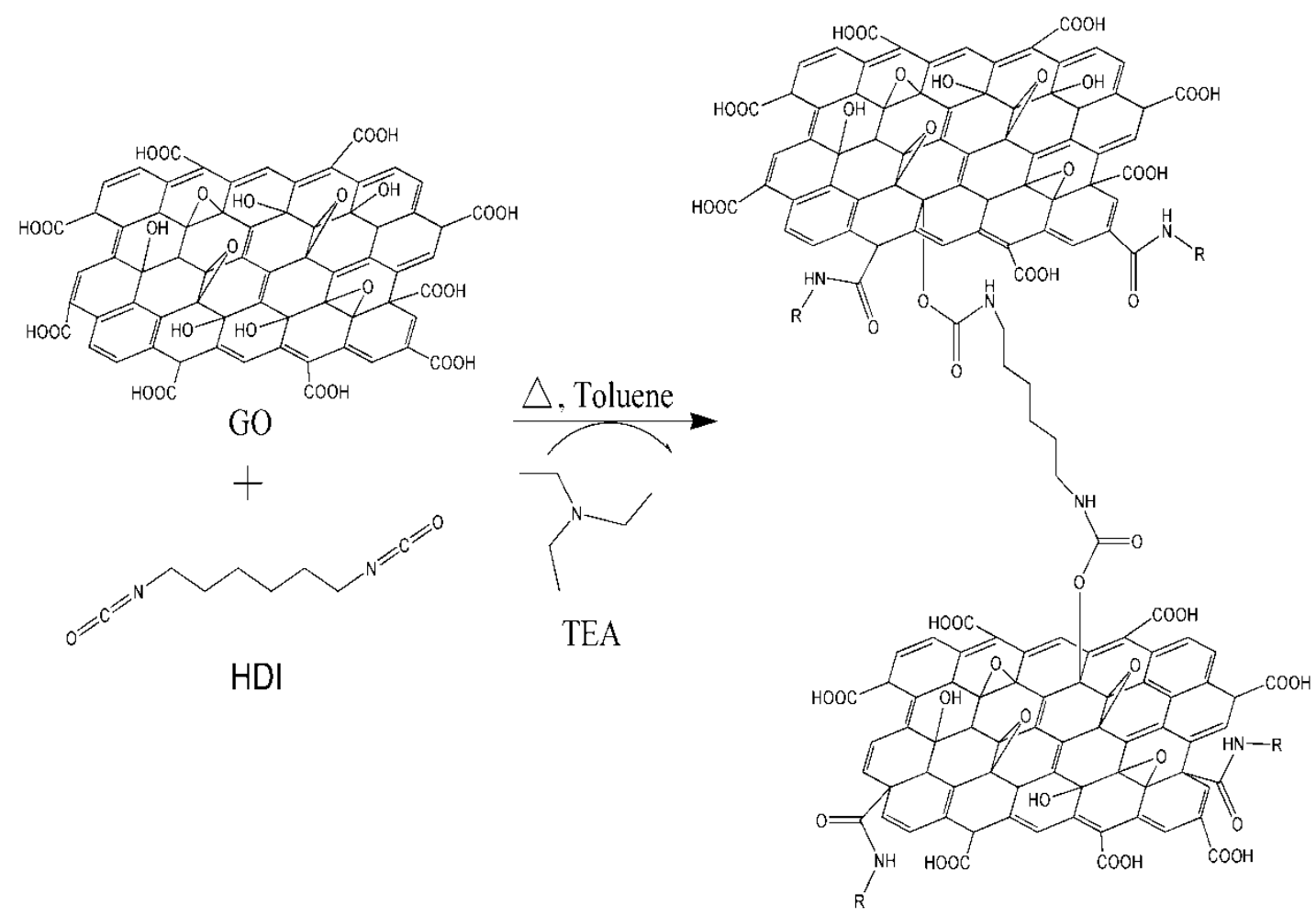

HDI-GO

Scheme 1. Schematic representation of the synthesis procedure of hexamethylene diisocyanate (HDI)functionalized graphene oxide (GO). 


\section{Results and Discussion}

The reaction conditions, namely reaction temperature, reaction time, GO/HDI/TEA ratio, tip/bath sonication cycles, and solvent volume were varied, in order to determine their effect on the product yield. The conditions of each experiment and nomenclature of the different HDI-GO samples obtained herein are detailed in Table 1.

Table 1. Nomenclature and reaction conditions for the synthesis of the different hexamethylene diisocyanate-graphene oxide (HDI-GO) samples.

\begin{tabular}{cccccc}
\hline Sample & $\begin{array}{c}\text { Reaction } \\
\text { Time (h) }\end{array}$ & $\begin{array}{c}\text { Reaction } \\
\text { Temperature } \\
\left({ }^{\circ} \mathbf{C}\right)\end{array}$ & $\begin{array}{c}\text { GO/HDI/Triethylamine } \\
\text { (TEA) Weight Ratio }\end{array}$ & $\begin{array}{c}\text { Tip/Bath } \\
\text { Sonication } \\
\text { Time (min) }\end{array}$ & $\begin{array}{c}\text { Solvent } \\
\text { Volume } \\
(\mathbf{m L})\end{array}$ \\
\hline GO & - & - & - & - & - \\
HDI-GO 1 & 12 & 60 & $1 / 1 / 1$ & $0 / 120$ & 25 \\
HDI-GO 2 & 12 & 60 & $0.5 / 1 / 1$ & $0 / 120$ & 25 \\
HDI-GO 3 & 48 & 60 & $1 / 1 / 1$ & $0 / 120$ & 25 \\
HDI-GO 4 & 12 & 90 & $1 / 1 / 1$ & $0 / 120$ & 25 \\
HDI-GO 5 & 12 & 60 & $1 / 1 / 1$ & $5 / 120$ & 50 \\
HDI-GO 6 & 12 & 60 & $1 / 1 / 1$ & $5+5+5^{*} / 120$ & 50 \\
\hline
\end{tabular}

* 3 probe sonication cycles with 5 min of break between cycles.

The chemical changes that occurred upon the treatment of GO with HDI were monitored by FTIR spectroscopy, since both GO and the derivatives display characteristic IR spectra (Figure 1). As mentioned earlier, GO contains epoxide and hydroxyl functional groups on both sides of its basal plane and carboxyl moieties at the edge sites. The most characteristic features in the IR spectrum of $\mathrm{GO}$ are the strong broad-band centred at $3430 \mathrm{~cm}^{-1}$, corresponding to the $\mathrm{O}-\mathrm{H}$ stretching vibrations, the peaks at around 2925 and $2845 \mathrm{~cm}^{-1}$ are attributed to sp2 and sp ${ }^{3} \mathrm{C}-\mathrm{H}$ stretching bands, produced at defects sites of the graphene network. The peak at $\sim 1730 \mathrm{~cm}^{-1}$ arising from the $\mathrm{C}=\mathrm{O}$ stretching of the carboxylic acid groups, the band at $1620 \mathrm{~cm}^{-1}$ assigned to the aromatic $\mathrm{C}-\mathrm{C}$ stretching, that at $\sim 1400 \mathrm{~cm}^{-1}$ corresponding to the $\mathrm{O}-\mathrm{H}$ deformation [11] and the $\mathrm{C}-\mathrm{OH}$ stretching at $1260 \mathrm{~cm}^{-1}$ [3].

Upon treatment with HDI, the intensity of the O-H stretching band was reduced; it decreased gradually with the increase in FD, and also shifted towards the lower wavenumbers, due to the overlapping with the N-H stretching vibrations of the carbamate groups. Further, the peaks at 2845 and $2925 \mathrm{~cm}^{-1}$, originating from the symmetrical and asymmetrical stretching vibrations of $-\mathrm{CH}_{2}$, became more intense as FD was raised, due to the increased number of methylene chains arising from the HDI. A new band appeared in the samples with low FD at $2280 \mathrm{~cm}^{-1}$ ascribed to unreacted $\mathrm{N}=\mathrm{C}=\mathrm{O}$ groups [12], suggestive of the absorption/intercalation of the organic isocyanate between the GO flakes. However, this band can hardly be detected in the HDI-GO 5 and HDI-GO 6 samples, corroborating that the $-\mathrm{N}=\mathrm{C}=\mathrm{O}$ groups of HDI had reacted completely with the hydroxyl groups of GO. Further, the $\mathrm{C}=\mathrm{O}$ stretching, appearing at $1730 \mathrm{~cm}^{-1}$ in pristine $\mathrm{GO}$, is hidden by a new peak at $\sim 1710 \mathrm{~cm}^{-1}$ ascribed to the $\mathrm{C}=\mathrm{O}$ stretching of the carbamate ester groups [10]. This peak becomes more intense and shifts gradually towards lower wave-numbers with increasing FD.

Further, new intense bands can be observed at $1648 \mathrm{~cm}^{-1}$ and $1580 \mathrm{~cm}^{-1}$; the first can be assigned to the coupling of the $\mathrm{C}=\mathrm{O}$ stretching with in-phase $\mathrm{N}-\mathrm{H}$ bending, and the second to the coupling of the $\mathrm{N}-\mathrm{H}$ bending with the $\mathrm{C}-\mathrm{N}$ stretching vibration [12]. These bands could originate from either amide or carbamate ester groups, although in the first case they typically appear at 1660 and 1550 $\mathrm{cm}^{-1}$, whilst for carbamate esters, the bands are closer together due to the stronger $\pi-\pi$ interaction between the carbonyl group and the nitrogen lone pair electrons, and the amide II band appears at a higher frequency [13]. These two peaks also turn out to be stronger and move to lower wave-numbers with increasing FD, which could be indicative of increased H-bonding interactions between carbamate esters that are closely located [14]. Other bands become visible at around $1110 \mathrm{~cm}^{-1}$, likely related to $-\mathrm{C}(=\mathrm{O})-\mathrm{O}$ and $\mathrm{C}-\mathrm{N}$ stretching vibrations of the carbamate groups, at $885 \mathrm{~cm}^{-1}$, attributed to $\mathrm{C}-\mathrm{H}$ out-of-plane bending vibrations of substituted aromatic rings, and at around $720 \mathrm{~cm}^{-1}$, 
ascribed to the rocking of the methylene groups of HDI. On the basis of all the aforementioned observations, it can be concluded that GO was successfully functionalized with the organic HDI reactant, and the functionalization route via carbamate ester formation predominates.

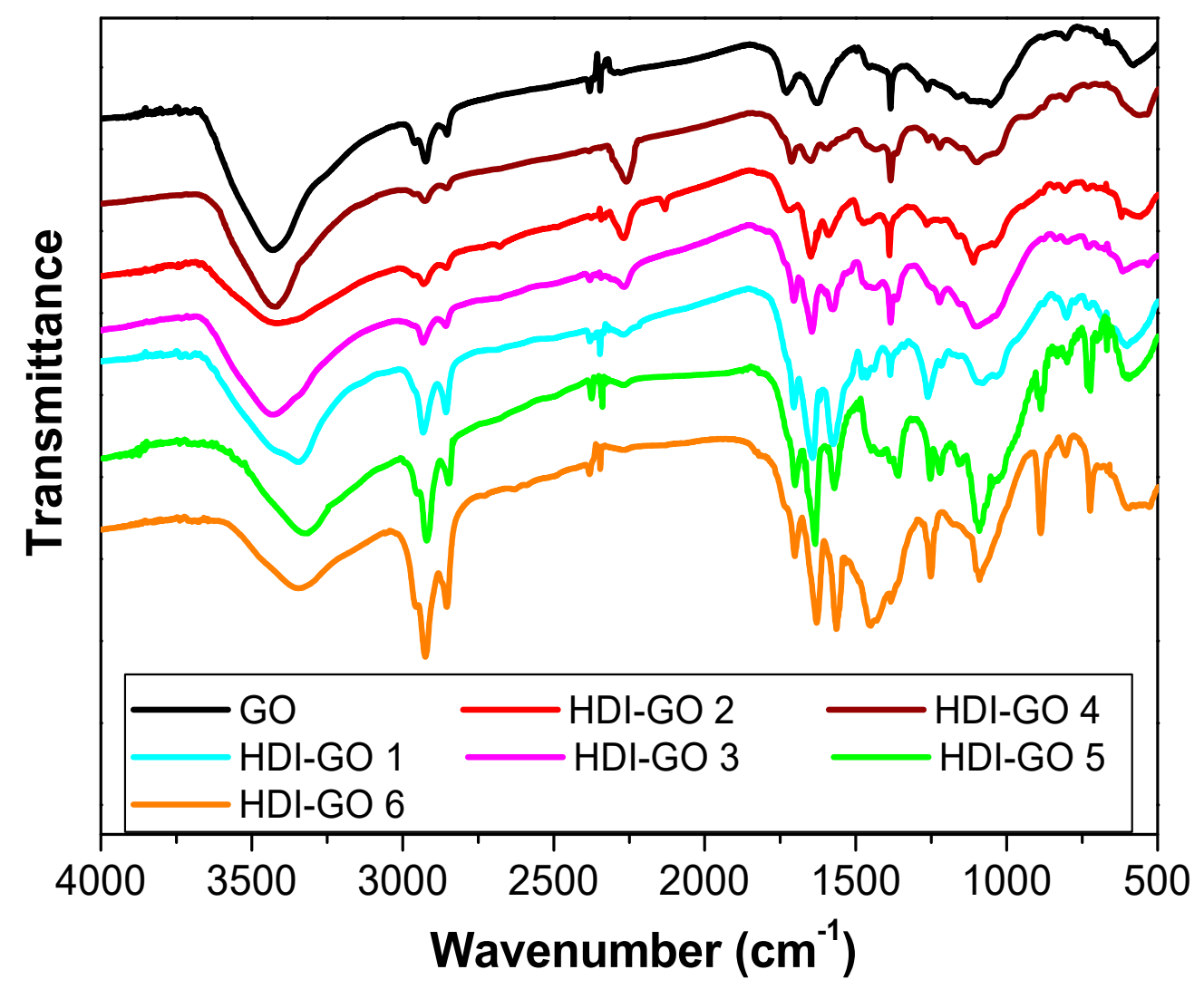

Figure 1. Fourier-transformed infrared (FT-IR) spectra of raw graphene oxide (GO) and the different hexamethylene diisocyante-functionalized graphene oxide (HDI-GO) samples.

\section{Materials and Methods}

\subsection{Reagents}

Natural graphite was obtained from Bay Carbon, Inc. (Bay City, MI, USA). $\mathrm{H}_{2} \mathrm{SO}_{4}, \mathrm{KMnO}_{4}, \mathrm{P}_{2} \mathrm{O}_{5}$, $\mathrm{K}_{2} \mathrm{~S}_{2} \mathrm{O}_{8}$, and $\mathrm{H}_{2} \mathrm{O}_{2}$ (30 wt \% in water) were purchased from Sigma-Aldrich and used as received. Hexamethylene diisocianate (HDI, $>99 \%, \mathrm{C}_{8} \mathrm{H}_{12} \mathrm{~N}_{2} \mathrm{O}_{2} \mathrm{M}_{\mathrm{w}}=168.196 \mathrm{~g} / \mathrm{mol}$ ) was acquired from Acros Organics. Triethylamine (TEA, $\left.>98 \%, \mathrm{~N}\left(\mathrm{CH}_{2} \mathrm{CH}_{3}\right)_{3}, \mathrm{M}_{\mathrm{w}}=101.193 \mathrm{~g} / \mathrm{mol}\right)$ was obtained from Fluka Analytical. All the organic solvents were high performance liquid chromatography (HPLC) grade and were purchased from Scharlau S.L. Toluene was dried and purified with a MBRAUN solvent purification system. Ultra-pure water was obtained from a Millipore Elix 15824 Advantage 15 UV purification system.

\subsection{Synthesis of Graphene Oxide (GO)}

GO was prepared using a modified Hummers' method from flake graphite [3,8]. Briefly, graphite powder, $\mathrm{H}_{2} \mathrm{SO}_{4}, \mathrm{~K}_{2} \mathrm{~S}_{2} \mathrm{O}_{8}$, and $\mathrm{P}_{2} \mathrm{O}_{5}$, were heated at $80{ }^{\circ} \mathrm{C}$ for $5 \mathrm{~h}$. After cooling, deionized water was added to the mixture and it was stirred overnight. The resulting product was then filtered, dried under air and oxidized again via addition of $\mathrm{H}_{2} \mathrm{SO}_{4}, \mathrm{KMnO}_{4}$ and water in an ice-water bath. Following to dilution with water, excess $\mathrm{KMnO}_{4}$ was decomposed by addition of 30 wt $\% \mathrm{H}_{2} \mathrm{O}_{2}$ aqueous solution and then $5 \mathrm{wt} \% \mathrm{HCl}$ aqueous solution. The product was filtered again and purified by repeating the following cycle: centrifugation, removal of the supernatant liquid, addition of aqueous solution of $\mathrm{H}_{2} \mathrm{SO}_{4}(3 \mathrm{wt} \%) / \mathrm{H}_{2} \mathrm{O}_{2}(0.5 \mathrm{wt} \%)$ and bath ultra-sonication for $30 \mathrm{~min}$ at a power 
of $140 \mathrm{~W}$. Then it was washed several times with deionized water and finally vacuum freeze-dried before use.

\subsection{Synthesis of Hexamethylene Diisocyanate-Functionalized Graphene Oxide (HDI-GO)}

The whole process was carried out under inert atmosphere of argon, in order to avoid contamination during the functionalization reaction. In a typical experiment, GO powder (c.a. 250 $\mathrm{mg}$ ) was weighed and loaded into a 100-mL round-bottom flask, followed by the addition of dried toluene $(25 \mathrm{~mL})$ under Ar atmosphere. The suspension was then ultra-sonicated in an ultrasonic bath for $2 \mathrm{~h}$; in some experiments, the bath sonication was preceded by probe sonication cycles $(5 \mathrm{~min} \mathrm{on} / 5$ min off, $40 \%$ amplitude). The sonication conditions were chosen according to preliminary studies carried out in the group [15]. The GO dispersion was then transferred to a reactor equipped with mechanical agitator, thermometer, and reflux condenser. Subsequently, TEA (c.a. $8.75 \mathrm{~mL}$ ) and HDI $(5 \mathrm{~mL})$ were added dropwise via a dropping funnel. The mixture was heated to $60{ }^{\circ} \mathrm{C}$ and stirred at $350 \mathrm{rpm}$ overnight under inert atmosphere. The resultant slurry reaction mixture was then poured into methylene chloride to coagulate the product, and finally filtered, washed thoroughly with methylene chloride, and dried under vacuum to yield HDI-GO. A schematic representation of the synthesis procedure of functionalized HDI-GO is shown in Scheme 1.

\subsection{Instrumentation}

FTIR spectra were recorded with a Perkin Elmer Frontier FTIR spectrophotometer equipped with an attenuated total reflection (ATR) sampling accessory. Spectra were recorded at room temperature, in the wavenumber range of $4000-500 \mathrm{~cm}^{-1}$, with an incident laser power of $1 \mathrm{~mW}$ and a minimum resolution of $4 \mathrm{~cm}^{-1}$. Prior to the measurements, the powder samples were mixed and ground with $\mathrm{KBr}$, and the mixtures were then pressed into a round transparent pellet in a pelletforming die.

\section{Conclusions}

Hexamethylene diisocyante-functionalized graphene oxide derivatives, with different functionalization degrees, have been synthesized following a two-step approach: Firstly, GO was prepared using a modified Hummers' method from graphite, and secondly GO was treated with HDI in the presence of TEA catalyst to yield the modified nanomaterial. The FT-IR and Raman spectra corroborated the success of the reaction, and the functionalization route via carbamate ester formation pre-dominated. Future work will focus on the reaction of HDI-GO with other organic molecules or polymers via the remaining oxygen groups, in order to develop high-performance GObased nanocomposites.

Author Contributions: A.M.D.-P. conceived and wrote the paper; C.G.-A. and G.M. designed the experiments, provided the reagents and the research facilities and contributed to the literature survey; J.A.L.-S. performed the experiments; A.M.D.-P. and J.A.L.-S. analyzed the data.

Acknowledgments: Financial support from Fundación Iberdrola España via a Research Grant in Energy and the Environment 2017 is gratefully acknowledged. A.M.D.-P would like to thank the Ministerio de Economía, Industria y Competitividad for a "Ramón y Cajal" post-doctoral fellowship.

Conflicts of Interest: The authors declare no conflict of interest.

\section{References}

1. Geim, A.K.; Novoselov, K.S. The rise of graphene. Nat. Mater. 2007, 6, 183-191, doi:10.1038/nmat1849.

2. Novoselov, K.S.; Geim, A.K.; Morozov, S.V.; Jiang, D.; Zhang, Y.; Dubonos, S.V.; Grigorieva, I.V.; Firsov, A.A. Electric field effect in atomically thin carbon films. Science 2004, 306, 666-669, doi:10.1126/science.1102896. 
3. Diez-Pascual, A.M.; Diez-Vicente, A.L. Poly(propylene fumarate)/polyethylene glycol-modified graphene oxide nanocomposites for tissue engineering. ACS Appl. Mater. Interfaces 2016, 8, 7902-17914, doi:10.1021/acsami.6b05635.

4. Díez-Pascual, A.M.; Gómez-Fatou, M.A.; Ania, F.; Flores, A. Nanoindentation in Polymer Nanocomposites. Prog. Mater. Sci. 2015, 67, 1-94, doi:10.1016/j.pmatsci.2014.06.002.

5. Salavagione, H.; Díez-Pascual, A.M.; Lázaro, E.; Vera, S.; Gomez-Fatou, M. Chemical sensors based on polymer composites with carbon nanotubes and G. The role of the polymer. J. Mater. Chem. 2014, 2, 1428914328, doi:10.1039/C4TA02159B.

6. Díez-Pascual, A.M., Luceño Sánchez, J.A., Peña Capilla, R., García Díaz, P. Recent advances in graphene/polymer nanocomposites for applications in polymer solar cells. Polymers 2018, 10, 217, doi:10.3390/polym10020217.

7. Aliotkhazraei, M.; Ali, N.; Milne, W.I.; Ozkan, C.S.; Mitura, S.; Gervasoni, J.L. Graphene Science Handbook: Fabrication Methods; CRC Press: Boca Raton, UK, 2016; ISBN 9781466591271.

8. Hummers, W.S.; Offeman, R.E. Preparation of Graphitic Oxide. J. Am. Chem. Soc. 1958, 80, 1339-1339, doi:10.1021/ja01539a017.

9. Titelman, G.I.; Gelman, V.; Bron, S.; Khalfin, R.L.; Cohen, Y.; Bianco-Peled, H. Characteristics and microstructure of aqueous colloidal dispersions of graphite oxide. Carbon 2005, 43, 641-649, doi:10.1016/j.carbon.2004.10.035.

10. Stankovich, S.; Piner, R.D.; Nguyen, S.T.; Rouff, R.S. Synthesis and exfoliation of isocyanate-treated graphene oxide nanoplatelets. Carbon 2006, 44, 3342-3347, doi:10.1016/j.carbon.2006.06.004.

11. Szabó, T.; Berkesi, O.; Dekany, I. DRIFT study of deuterium-exchanged graphite oxide. Carbon 2005, 43, 3186-3189, doi:10.1016/j.carbon.2005.07.013.

12. Lin-Vien, D.; Colthup, N.; Fateley, W.; Grasselli, J. The Handbook of Infrared and Raman Characteristic Frequencies of Organic molecules. Academic Press: San Diego, United States, 1991, ISBN 9780080571164.

13. Cannon, C.G. Infrared frequencies of amide, urea, and urethane groups. J. Phys. Chem. 1976, 80, 1247-1248, doi:10.1021/j100552a026.

14. Díez-Pascual, A.M.; Díez-Vicente, A.L. Poly(3-hydroxybutyrate)/ZnO Bionanocomposites with Improved Mechanical, Barrier and Antibacterial Properties. Int. J. Mol. Sci. 2014, 15, 10950-10973, doi:10.3390/ijms150610950.

15. Díez-Pascual, A.M.; García-García, D.; San Andrés, M.P.; Vera, S. Determination of riboflavin based on fluorescence quenching by graphene dispersions in polyethylene glycol. RSC Adv. 2016, 6, 19686-19699. doi:10.1039/c5ra25547c.

(C) 2018 by the authors. Licensee MDPI, Basel, Switzerland. This article is an open access article distributed under the terms and conditions of the Creative Commons Attribution (CC BY) license (http://creativecommons.org/licenses/by/4.0/). 